\title{
Resultados pressóricos da esclerectomia profunda não penetrante no tratamento do glaucoma primário de ângulo aberto
}

\author{
Tensional resultsof non-penetrating deep sclerectomy in the treatment of primary \\ open-angle glaucoma
}

Ricardo Augusto Paletta Guedes ${ }^{1}$ Vanessa Maria Paletta Guedes ${ }^{2}$

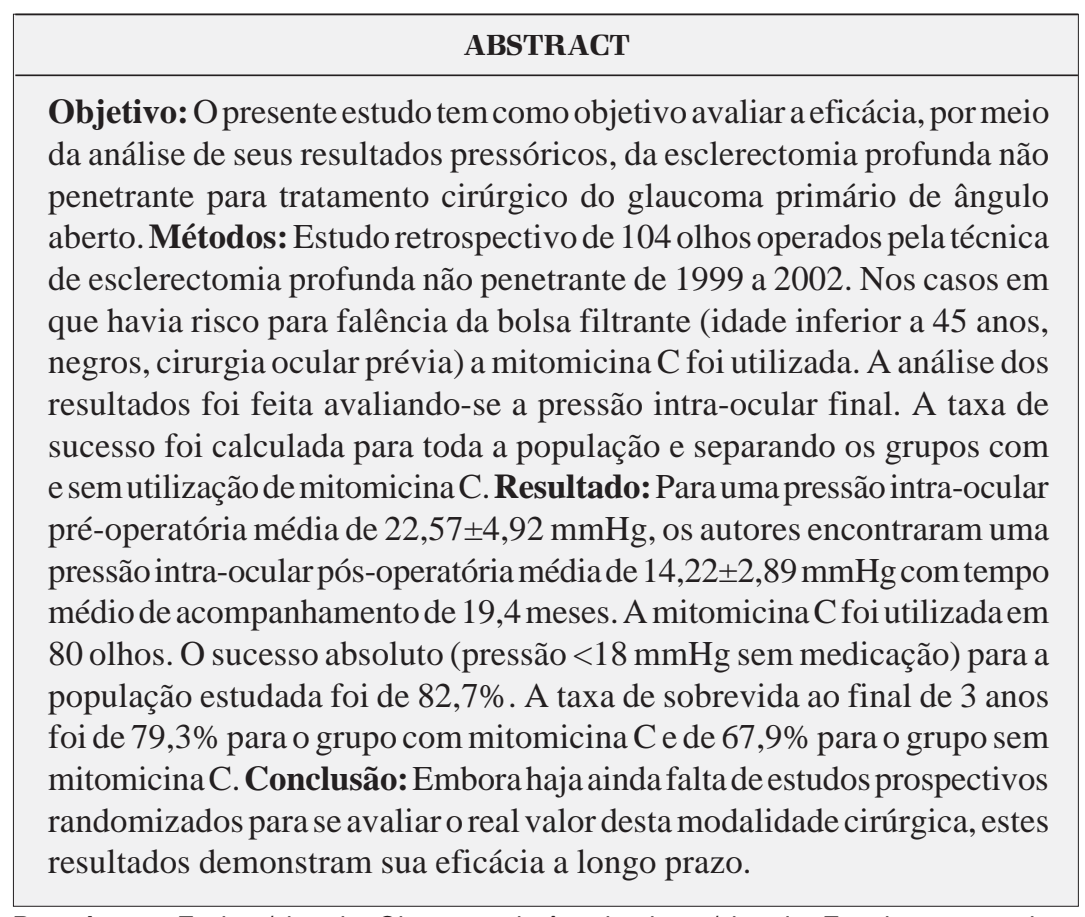

Descritores: Esclera/cirurgia; Glaucoma de ângulo aberto/cirurgia; Estudos prospectivos

\section{INTRODUÇÃO}

O glaucoma primário de ângulo aberto consiste em uma doença multifatorial caracterizada por uma lesão progressiva do disco óptico, causando uma alteração do campo visual, podendo levar a cegueira irreversível. O aumento da pressão intra-ocular (Po) é o principal fator de risco para a alteração no nervo óptico ${ }^{(1)}$.

Acredita-se que o aumento da pressão intra-ocular no glaucoma primário de ângulo aberto seja por uma obstrução da via de escoamento do humor aquoso, mais precisamente no trabeculado justacanalicular e parede interna do canal de Schlemm ${ }^{(2)}$.

O tratamento cirúrgico do glaucoma, quando indicado, tem como objetivo a diminuição da Po a valores que permitam impedir a evolução do dano glaucomatoso ou mesmo evitá-lo. Esta é a chamada Po-alvo ${ }^{(3)}$.

A trabeculectomia é a técnica cirúrgica mais utilizada no mundo, apresentando resultados satisfatórios, porém com índice razoável de complica- 
ções per e pós-operatórias. No momento, na Europa, principalmente na França, as técnicas não penetrantes da cirurgia do glaucoma tendem a substituir a trabeculectomia convencional. Algumas vantagens apresentadas pela cirurgia não penetrante incluem: possibilidade de realização em pacientes com dano avançado/terminal de campo visual; baixa taxas de complicações per e pós-operatórias e manutenção da acuidade visual pré-operatória, o que torna o acompanhamento pós-operatório mais simples para o médico e para o paciente ${ }^{(4)}$.

O objetivo do presente estudo é de se avaliar a eficácia, em termos de diminuição da Po, da esclerectomia profunda não penetrante no tratamento cirúrgico do glaucoma primário de ângulo aberto.

\section{MÉTODO}

Os autores avaliaram retrospectivamente 104 olhos submetidos a cirurgia não penetrante pela técnica de "esclerectomia profunda não penetrante com trabeculectomia externa” de 1999 a 2002.

Todos os olhos possuíam glaucoma primário de ângulo aberto: alteração típica do disco óptico e alteração típica do campo visual. O campo visual foi classificado em precoce, moderado ou severo de acordo com a classificação de Anderson ${ }^{(5)}$. A indicação cirúrgica foi feita quando havia um insuficiente controle clínico ou uma indicação social (paciente sem condições econômicas de comprar a medicação hipotensora).

A técnica cirúrgica utilizada foi a seguinte:

1. Tração corneana com vycril 7.0;

2. Abertura da conjuntiva e cápsula de Tenon com base fórnice;

3. Esponja com mitomicina C $0,2 \mathrm{mg} / \mathrm{ml}$ por 3 minutos, quando indicado (pacientes jovens < 45 anos; negros; cirurgia ocular prévia), seguida de lavagem abundante com soro fisiológico;

4. Confecção do retalho superficial escleral quadrangular de 5 x 5 mm com 1/3 de profundidade;

5. Confecção do retalho profundo escleral triangular de base de 4 a 5 mm até o plano pré-ciliar;

6. Excisão do retalho profundo, retirando junto à parede externa do canal de Schlemm;

7. Excisão da membrana trabecular externa, que inclui a parede interna do canal e o trabeculado justacanalicular;

8. Sutura do retalho superficial com 1 ponto frouxo de mononylon 10.0 ;

9. Sutura da conjuntiva por pontos separados de vycril 7.0.

Os pacientes foram examinados com 1, 7, 15, 30, 60, 90, 180, 360, 540, 720, 900, 1080 dias de pós-operatório e a cada consulta era realizada acuidade visual, biomicroscopia de segmento anterior e do disco óptico, tonometria de aplanação. Um campo visual era realizado de 6 em 6 meses (Humphrey 24-2 Fullthreshhold). Uma gonioscopia era realizada sempre que necessário (casos em que havia um aumento da pressão intra- ocular pós-operatório). O número médio de colírios no préoperatório foi comparado ao do pós-operatório.

Foi observada a performance desta técnica no plano tonométrico e realizado o cálculo das taxas de sucesso:

- Sucesso absoluto: Po <18 mmHg sem medicação hipotensora ocular;

- Sucesso relativo: Po <18 mmHg com medicação hipotensora ocular local.

As curvas de sobrevida com probabilidade de sucesso foram calculadas para a população toda e para os grupos em separado pelo método de Kapplan-Meier (Po <18 mmHg sem medicação hipotensora).

\section{RESULTADOS}

A população estudada era composta de 55,8\% de homens e de $44,2 \%$ de mulheres; de $57,7 \%$ de caucasianos e de $42,3 \%$ de pacientes da raça negra. A mitomicina $\mathrm{C}$ foi utilizada em 80 olhos.

O tempo de acompanhamento médio foi de 19,42 meses (mínimo de 2 e máximo de 36 meses).

Para uma Po pré-operatória média de 22,57£7,92 mmHg, os autores encontraram uma Po média ao final do acompanhamento de 14,22 22,89 mmHg. As comparações pré e pós-operatória para a população total estão demonstradas na tabela 1. Os resultados pertinentes aos grupos com e sem utilização de mitomicina C estão expostos nas tabelas 2 e 3, respectivamente.

\section{Tabela 1. Comparação pré e pós-operatória}

\begin{tabular}{|c|c|c|}
\hline \multicolumn{2}{|c|}{ Po pré-operatória } & $22,57 \pm 4,92 \mathrm{mmHg}$ \\
\hline \multicolumn{3}{|c|}{ Po pós-operatória: } \\
\hline D1 & $(\mathrm{N}=104)$ & $6,56 \pm 3,22 \mathrm{mmHg}$ \\
\hline D7 & $(\mathrm{N}=104)$ & $8,65 \pm 3,08 \mathrm{mmHg}$ \\
\hline D15 & $(\mathrm{N}=104)$ & $10,15 \pm 3,70 \mathrm{mmHg}$ \\
\hline D30 & $(\mathrm{N}=104)$ & $11,82 \pm 3,49 \mathrm{mmHg}$ \\
\hline D60 & $(\mathrm{N}=104)$ & $12,81 \pm 3,57 \mathrm{mmHg}$ \\
\hline D90 & $(\mathrm{N}=101)$ & $13,34 \pm 4,09 \mathrm{mmHg}$ \\
\hline D180 & $(\mathrm{N}=91)$ & $14,09 \pm 4,17 \mathrm{mmHg}$ \\
\hline D360 & $(\mathrm{N}=80)$ & $14,45 \pm 3,26 \mathrm{mmHg}$ \\
\hline D540 & $(\mathrm{N}=60)$ & $14,47 \pm 2,98 \mathrm{mmHg}$ \\
\hline D720 & $(\mathrm{N}=44)$ & $14,27 \pm 3,12 \mathrm{mmHg}$ \\
\hline D900 & $(\mathrm{N}=35)$ & $14,03 \pm 2,24 \mathrm{mmHg}$ \\
\hline D108C & $(\mathrm{N}=18)$ & $15,89 \pm 2,91 \mathrm{mmHg}$ \\
\hline AV pré-o & seratória & $0,6078 \pm 0,2957$ \\
\hline AV pós-o & peratória & $0,5673 \pm 0,2835$ \\
\hline C/D pré-c & peratória & $0,740 \pm 0,146$ \\
\hline C/D pós- & operatória & $0,753 \pm 0,145$ \\
\hline Número c & le colírios pré-operatório & $2,48 \pm 0,79$ \\
\hline Número c & le colírios pós-operatório & $0,33 \pm 0,74$ \\
\hline $\begin{array}{l}\text { CV pré-o } \\
\text { precoce/r }\end{array}$ & $\begin{array}{l}\text { peratório } \\
\text { noderado/severo }\end{array}$ & $12,5 \% / 38,5 \% / 49,0 \%$ \\
\hline $\begin{array}{l}\text { CV pós-o } \\
\text { precoce/r }\end{array}$ & $\begin{array}{l}\text { peratório } \\
\text { noderado/severo }\end{array}$ & $12,5 \% / 36,5 \% / 51,0 \%$ \\
\hline $\begin{array}{l}\text { Po: pressã } \\
\text { vertical da }\end{array}$ & $\begin{array}{l}\text { o intra ocular; D: dia; AV: acu } \\
\text { escavação/diâmetro vertical }\end{array}$ & $\begin{array}{l}\text { visual; C/D: relação diâmetro } \\
\text { ila; CV: campo visual }\end{array}$ \\
\hline
\end{tabular}




\begin{tabular}{|c|c|c|c|}
\hline & $\mathbf{N}$ & Média & Desvio padrão \\
\hline $\begin{array}{l}\text { Número de colírios } \\
\text { pré-operatório }\end{array}$ & 80 & 2,48 & 0,81 \\
\hline C/D pré-operatório & 80 & 0,752 & 0,147 \\
\hline AV pré-operatória & 80 & 0,6186 & 0,2943 \\
\hline Po pré-operatória & 80 & 22,59 & 5,18 \\
\hline Po Dia 1 & 80 & 6,48 & 3,46 \\
\hline Po Dia 7 & 80 & 8,66 & 3,21 \\
\hline Po Dia 15 & 80 & 9,91 & 3,59 \\
\hline Po Dia 30 & 80 & 11,58 & 3,45 \\
\hline Po Dia 60 & 80 & 12,41 & 2,86 \\
\hline Po Dia 90 & 77 & 13,06 & 4,14 \\
\hline Po Dia 180 & 71 & 13,86 & 4,61 \\
\hline Po Dia 360 & 61 & 14,38 & 3,40 \\
\hline Po Dia 540 & 46 & 14,35 & 2,88 \\
\hline Po Dia 720 & 35 & 14,57 & 2,85 \\
\hline Po Dia 900 & 28 & 14,14 & 2,35 \\
\hline Po Dia 1080 & 13 & 16,46 & 2,96 \\
\hline Po final & 80 & 14,15 & 2,93 \\
\hline $\begin{array}{l}\text { Número colírios } \\
\text { pós-operatório }\end{array}$ & 80 & 0,31 & 0,72 \\
\hline $\mathrm{C} / \mathrm{D}$ final & 80 & 0,762 & 0,148 \\
\hline AV final & 80 & 0,5846 & 0,2779 \\
\hline \multicolumn{4}{|c|}{$\begin{array}{l}\mathrm{N} \text { : número de pacientes; C/D: relação diâmetro vertical da escavação/diâmetro } \\
\text { vertical da papila; AV: acuidade visual; Po: pressão intra-ocular }\end{array}$} \\
\hline
\end{tabular}

A taxa de sucesso absoluto foi de $82,7 \%$ para o grupo total de 104 olhos; 83,3\% para grupo de 24 olhos sem utilização de mitomicina C; e 82,5\% para o grupo de 80 olhos com utilização de mitomicina $\mathrm{C}$ no per-operatório.

A taxa de sucesso relativa foi de $93,3 \%$ para o grupo total; $91,7 \%$ para o grupo sem mitomicina C e $93,8 \%$ para o grupo com mitomicina $\mathrm{C}$.

As curvas de probabilidade de sucesso são representadas nos gráficos 1 e 2 , tendo uma probabilidade de sucesso ao final de 1080 dias de 76,1\% para a população estudada e de $67,9 \%$ e $79,3 \%$ para os grupos sem e com mitomicina C, respectivamente.

As complicações são objeto de outro estudo dos mesmos autores.

\section{DISCUSSÃO}

O melhor conhecimento fisiopatológico do glaucoma primário de ângulo aberto (GPAA) e a precisão histológica permitiram a evolução da técnica da cirurgia filtrante do glaucoma ${ }^{(6)}$.

A esclerectomia profunda não penetrante com trabeculectomia externa aparece como uma boa alternativa para o tratamento cirúrgico do GPAA ${ }^{(6)}$.

O precursor da cirurgia não penetrante é sem dúvida o cirurgião russo M. M. Krasnov, que inventa em 1964 uma intervenção que ele batiza sinusotomia. Nos anos 70, o professor Fyodorov, aproveitando a idéia de Krasnov de uma resistência intraescleral ao escoamento, propõe uma outra intervenção: a esclerectomia profunda não penetrante. Ela consiste na abertu-

\begin{tabular}{|lccc|}
\hline \multicolumn{4}{|c|}{ Tabela 3. Resultados para o grupo de $\mathbf{2 4}$ olhos sem mitomicina C } \\
Número de colírios & $\mathbf{N}$ & Média & Desvio padrão \\
pré-operatório & 24 & 2,50 & 0,72 \\
C/D pré-operatório & 24 & 0,700 & 0,141 \\
AV pré-operatória & 24 & 0,5727 & 0,3044 \\
Po pré-operatória & 24 & 22,50 & 4,03 \\
Po Dia 1 & 24 & 6,83 & 2,28 \\
Po Dia 7 & 24 & 8,63 & 2,67 \\
Po Dia 15 & 24 & 10,96 & 4,01 \\
Po Dia 30 & 24 & 12,63 & 3,55 \\
Po Dia 60 & 24 & 14,13 & 5,15 \\
Po Dia 90 & 24 & 14,21 & 3,89 \\
Po Dia 180 & 20 & 14,90 & 1,74 \\
Po Dia 360 & 19 & 14,68 & 2,81 \\
Po Dia 540 & 14 & 14,86 & 3,35 \\
Po Dia 720 & 9 & 13,11 & 3,98 \\
Po Dia 900 & 7 & 13,57 & 1,81 \\
Po Dia 1080 & 5 & 14,40 & 2,41 \\
Po final média & 24 & 14,46 & 2,81 \\
Número colírios & 24 & 0,38 & 0,82 \\
pós-operatório & & & \\
C/D final & 24 & 0,721 & 0,135 \\
AV final & 24 & 0,5082 & 0,3015 \\
N: número de pacientes; C/D: relação diâmetrovertical da escavação/diâmetro \\
vertical da papila; AV: acuidade visual; Po: pressão intra-ocular \\
\hline
\end{tabular}

ra do canal de Schlemm (sinusotomia) pela via posterior, associada à excisão de um retalho escleral profundo pré-ciliar. Neste ponto, duas escolas se divergem: uma que preconiza abertura do canal e em seguida a excisão da parede interna do canal juntamente com o trabéculo cribriforme ou justacanalicular (Zimmermann, 1977); outra que propõe a abertura do canal seguida de uma dissecção profunda até a córnea na procura de uma filtração através da membrana de Descemet ${ }^{(7)}$.

A esclerectomia profunda não penetrante foi introduzida na Europa ocidental por Demailly em $1993^{(8)}$. Na tentativa de se melhorar a filtração do humor aquoso, Demailly, seguindo o exemplo de Koslov et al. ${ }^{(9)}$, utilizou um implante de colágeno no leito escleral profundo. Seus resultados não mostraram diferenças comparando-se o implante de colágeno com a aplicação de 5-fluoro-uracil(8). Em estudo prospectivo recente, verificou-se que a utilização per-operatória de mitomicina C no leito escleral aumenta a chance de sucesso deste procedimento cirúrgico ${ }^{(10)}$.

Recentemente foi feita uma revisão das técnicas cirúrgicas não penetrantes muito interessante ${ }^{(4)}$. As três etapas mais importantes são:

1. Confecção do retalho superficial escleral;

2. Confecção do retalho profundo escleral evidenciando o canal de Schlemm;

3. Excisão da membrana trabecular externa, compreendendo a parede interna do canal e o trabeculado justacanalicular ${ }^{(6)}$. Estudos em microscopia confocal da membrana retirada durante o procedimento cirúrgico demonstraram que ela com- 


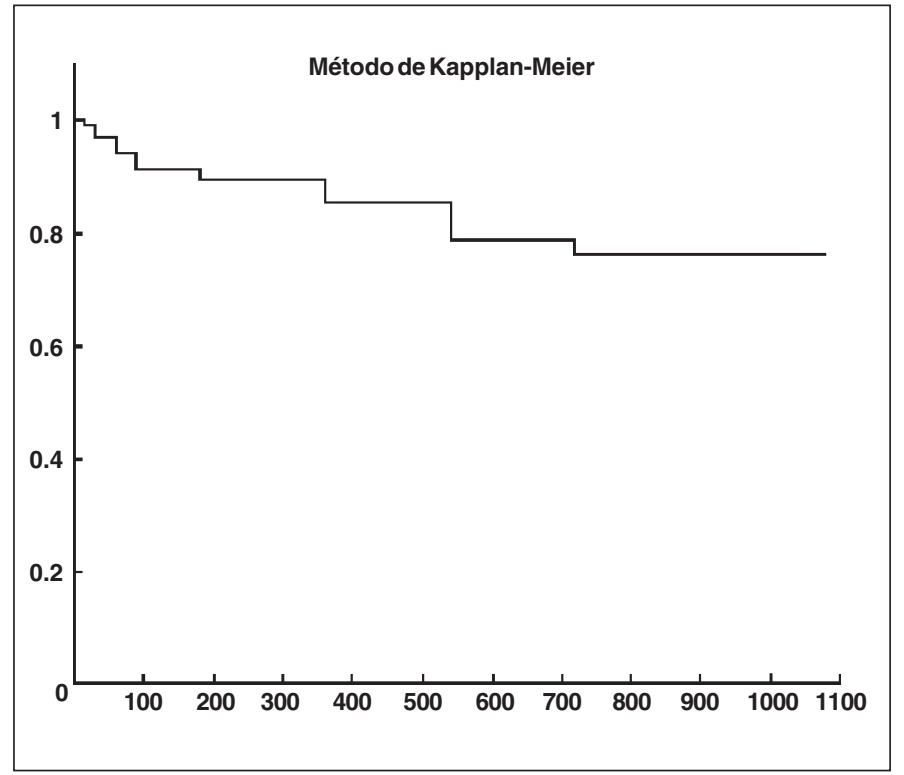

Gráfico 1 - Probabilidade de sucesso (104 olhos) após 1080 dias: 0,761

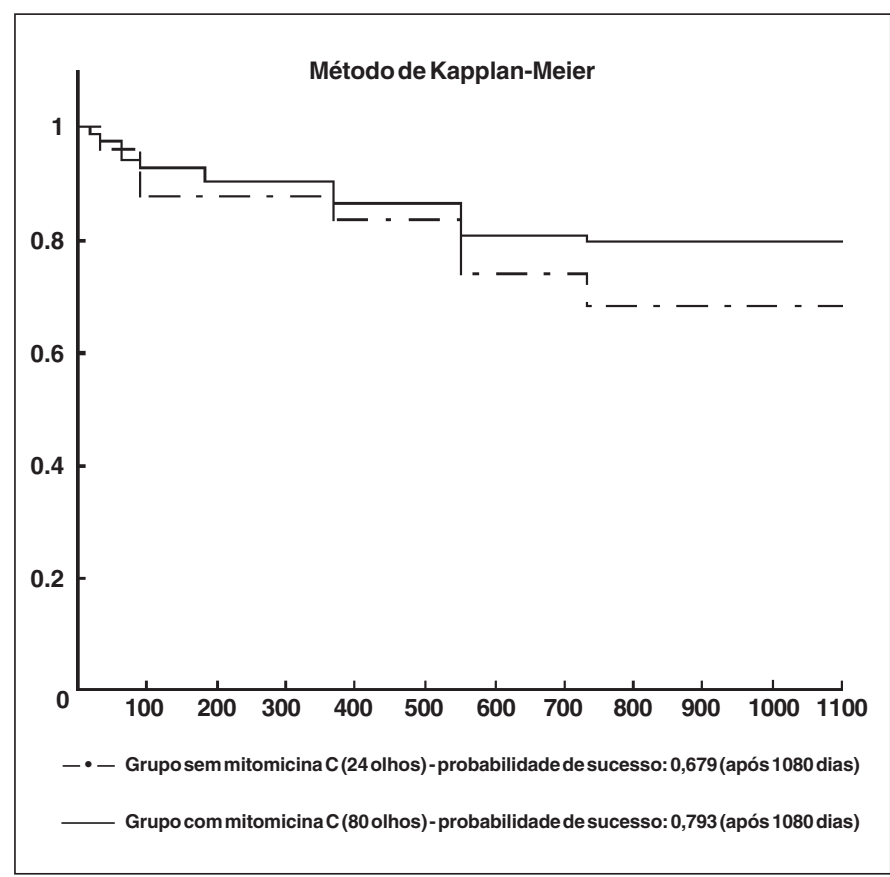

Gráfico 2 - Probabilidade de sucesso para os diferentes grupos

preendia: a parede interna do canal de Schlemm; o trabeculado cribriforme e parte do trabeculado corneoescleral. Ela tinha uma espessura constante de 25 a 30 micra. Esta região é a responsável pela maior resistência ao escoamento do humor aquoso em olhos glaucomatosos ${ }^{(11)}$.

A esclerectomia profunda não penetrante mostrou-se eficaz na redução pressória em pacientes portadores de GPAA em diversos estudos ${ }^{(4)}$. A Po-alvo é um conceito novo que deve ser levado em consideração no tratamento do glaucoma.
Estudos recentes tem demonstrado que pacientes que mantém a Po abaixo de $18 \mathrm{mmHg}$ a longo prazo em todas as medidas tem uma chance muito diminuída de evolução do dano glaucomatoso $^{(12)}$. Por isto, os autores escolheram o valor de $18 \mathrm{mmHg}$ para as taxas de sucesso calculadas no presente estudo.

Os resultados pressóricos encontrados pelos autores são comparáveis aos estudos publicados anteriormente ${ }^{(4)}$. Para uma Po pré-operatória média de 22,57 44,92 mmHg, foi encontrada uma Po média final de 14,22 $\pm 2,89$ mmHg (acompanhamento médio de 19,42 meses). A Po abaixou bastante no primeiro mês de pós-operatório ( $<12 \mathrm{mmHg}$ ) e, depois houve uma estabilização em torno de $14 \mathrm{mmHg}$ (de 3 meses até 30 meses). O número de colírios hipotensores diminuiu de 2,48 $\pm 0,79$ a $0,33 \pm 0,74$ em média, reduzindo o custo do tratamento. Isto é particularmente muito importante para os pacientes com problemas socioeconômicos em nosso país.

A taxa de sucesso apresentada aqui é comparável aos principais estudos na literatura. Para uma $\mathrm{PIO}<21 \mathrm{mmHg}$ sem tratamento, Massy encontrou uma taxa de $81 \%$ a 14,2 meses; Mermoud, 69\% a 14,4 meses; Demailly, 68\%-69\% a 20 meses; Lachkar, $75,7 \%-75 \%$ a 60 meses; Hamard, 57,3\%-66\% a 11 meses; Bas, $92 \%$ a 5,3 meses ${ }^{(4)}$.

Os autores encontraram uma taxa de sucesso absoluto (PIO $<18$ mmHg sem medicação) de $82,7 \%$ e de sucesso relativo (PIO $<18 \mathrm{mmHg}$ com hipotensores oculares) de 93,3\% para um tempo médio de acompanhamento de 19,4 meses. A taxa de sobrevida, medida pela curva de Kapplan-Meier, foi de 76,1\% ao final de 3 anos para a população global, mostrando uma boa eficácia a longo prazo para um procedimento filtrante. Esta mesma taxa mostrou-se diferente quando a análise é feita para os grupos separadamente. O grupo com utilização per-operatória de mitomicina $\mathrm{C}$ apresentou uma taxa de sucesso ao final de 3 anos de $79,3 \%$ contra $67,9 \%$ para o grupo sem mitomicina $\mathrm{C}$, reforçando o achado de que a mitomicina C aumenta o índice de sucesso da cirurgia ${ }^{(10)}$. Este estudo confirma os resultados preliminares dos mesmos autores no que diz respeito à eficácia desta técnica cirúrgica para a população brasileira $^{(13)}$.

Existe uma falta de estudos prospectivos randomizados com grande número de pacientes que confirmem a verdadeira importância desta modalidade cirúrgica do tratamento do GPAA, porém estudos retrospectivos como este demonstram indubitavelmente que esta técnica deve ser levada a sério e que ela começa a mostrar que tem seu lugar no arsenal terapêutico cirúrgico desta doença que permanece sendo um problema de saúde pública no Brasil.

\section{CONCLUSÃO}

A esclerectomia profunda não penetrante com trabeculectomia externa mostrou-se eficaz no controle pressórico do GPAA até 3 anos de acompanhamento. 


\section{ABSTRACT}

Purpose: The aim of this study is to assess the efficacy, of the non-penetrating procedure for surgical treatment of primary open-angle glaucoma, by the analysis of its tensional results. Methods: Retrospective study of 104 eyes with primary openangle glaucoma submitted to a non-penetrating deep sclerectomy from 1999 to 2002. Mitomycin C was used in cases with high risk for bleb failure, such as, age under 45 years, blacks, previous ocular surgery. Final mean intraocular pressure was observed and success rate was calculated for the whole population and for each group (with and without mitomycin C). Results: For a mean preoperative intraocular pressure of $22.57 \pm 4.92 \mathrm{mmHg}$, the authors found a mean final intraocular pressure of $14.22 \pm 2.89 \mathrm{mmHg}$. Mitomycin C was used in 80 eyes. Absolute success (intraocular pressure $<18 \mathrm{mmHg}$ without medication) for the entire group was $82.7 \%$, with a follow-up of 19.4 months on average. Success rate after 3 years of follow-up, by the Kapplan-Meier method, was 79.3\% for the mitomycin $\mathrm{C}$ group and $67.9 \%$ for the other group. Conclusion: Although there is a lack of prospective randomized studies in order to evaluate the real value of this recent procedure, these results demonstrate its efficacy in a longterm follow-up.

Keywords: Sclera/surgery; Glaucoma, open-angle/surgery; Prospective studies

REFERÊNCIAS

1. Shields MB. Textbook of glaucoma. $4^{\mathrm{a}}$ ed. Baltimore: Williams \& Wilkins; 1998.

2. Béchetoille A. Les glaucomes. Angers: Japperenard; 1997.

3. Bron A, Béchetoille A. La pression cible. Visions Internationales 1998; (November):3-4.

4. Hamard P, Lachkar Y. La chirurgie filtrante non perforante: évolution du concept, réalisation, résultats. J Fr Ophtalmol. 2002;25(5):527-36.

5. Andreson DR, Patella, VM. Automated Static Perimetry. St Louis: Mosby; 1999.

6. Bove G, Kantelip B, Algros MP. La sclérectomie profonde avec trabéculectomie externe: un formidable pari! J Fr Ophtalmol. 2001;24(3):332-3.

7. Valtot F. La chirurgie non perforante du glaucome: les différentes technique et leurs modes d'action. In: Béchetoille A. editors. $7^{\text {e }}$ Symposium Recherche et Glaucome. Orlando; 1999. p.75-84.

8. Demailly P, Jeanteur-Lunel MN, Berkani M, Ecoffet M, Kopel J, Kretz G, Lavat P. La sclérectomie profonde non perforante associée à la pose d'un implant de collagène dans le glaucome primitif à angle ouvert. Résultats rétrospectifs à moyen terme. J Fr Ophtalmol. 1996;19(11):659-66.

9. Koslov VI et al. Non penetrating deep sclerectomy with collagen implant. IRTC Eye Microsurgery. Moscow: RSFSR Ministry of Public Health; 1990. p. 44-6 apud Mermoud A: La sclérectomie profonde. Technique chirurgicale. J Fr Ophtalmol. 1999;22(7):781-6.

10. Kozobolis VP, Christodoulakis EV, Tzanakis N, Zacharopoulos I, Pallikaris IG. Primary deep sclerectomy versus primary deep sclerectomy with the use of mitomycin C in primary open-angle glaucoma. J Glaucoma. 2002;11(4):287-93.

11. Hamard P, Sourdille P, Valtot F, Baudouin C. Apport de la microscopie confocale dans l'analyse de la membrane trabéculaire prélevée au cours de la chirurgie filtrante non perforante. J Fr Ophtalmol. 2001;24(1):29-35.

12. The Advanced Glaucoma Intervention Study (AGIS): 7. The relationship between control of intraocular pressure and visual field deterioration. The AGIS Investigators. Am J Ophthalmol. 2000;130(4):429-40.

13. Guedes VMP, Guedes RAP. Esclerectomia profunda não perfurante : resultados a médio prazo dos primeiros pacientes operados. Rev Bras Oftalmol 2001; 60(1):20-4.

\title{
XXV CONGRESSO DO HOSPITAL SÃO GERALDO
}

\section{1 a 13 de novembro de 2004 Belo Horizonte - MC}

\author{
INFORMAC̣ÕES: Consult Comunicação e Eventos \\ Fone/Fax: (31) 3274-1550 \\ E-mail: comunica@consultcom.com.br
}

